\title{
Stimulating Local Public Employment: Do General Grants Work?
}

\author{
Heléne Lundqvist $\quad$ Matz Dahlberg Eva Mörk \\ Online Appendix
}

This Appendix includes supplementary material to Lundqvist, Dahlberg and Mörk: "Stimulating Local Public Employment: Do General Grants Work?"

A few comments:

- Table 1: First-stage estimates when controlling for pre-determined covariates.

- Table 2: First-stage estimates when controlling for municipality fixed effects.

- Table 3: Second-stage estimates when controlling for municipality fixed effects.

- Table 4: First-stage estimates with the shorter panel-i.e., only years 200204 for which we have data on the number of employed in the outsourced parts of child care, schools, elderly care and social welfare. Due to the large sample reduction, the significance of the first stage is sensitive to order of polynomial and bandwidth. Therefore, we only estimate the second stage using the three widest bandwidths while controlling for out-migration linearly.

- Table 5: Effects of grants on municipal personnel using the shorter panel. That is, the main results on public employment, but only for years 2002-04.

- Table 6: Effects of grants on personnel in the local public welfare sector employed by a non-profit or for-profit private firm. Results obtained with data covering 2002-04.

- Table 7: Effects of grants on private school personnel. Here data covers the entire period 1996-2004, and this table presents analysis for school personnel employed by a non-profit or for-profit private firm. Since this is the full sample period and the first stage therefore is less sensitive to order of polynomial and bandwidth, various alternatives of the second stage is estimated. 
Table 1: First-stage estimates when controlling for predetermined covariates.

\begin{tabular}{lcccc}
\hline & Full sample & $h=15$ & $h=10$ & $h=5$ \\
\hline $\bar{p}=1$ & $4.429^{* * *}$ & $4.013^{* * *}$ & $4.348^{* * *}$ & $2.738^{* * *}$ \\
& $(0.615)$ & $(0.659)$ & $(0.696)$ & $(0.840)$ \\
\hline $\bar{p}=2$ & $3.777^{* * *}$ & $3.653^{* * *}$ & & \\
& $(0.687)$ & $(0.993)$ & & \\
\hline $\bar{p}=3$ & $3.791^{* * *}$ & $4.473^{* * *}$ & & \\
& $(0.979)$ & $(1.234)$ & & \\
\hline Observations & 2511 & 2346 & 2047 & 1241 \\
\hline
\end{tabular}

Note: For different bandwidths, $h$, and order of polynomials, $\bar{p}$, the table reports estimates of $\alpha_{1}$ in the first-stage equation (3) on cost-equalizing grants, when controlling for pre-determined covariates. Standard errors clustered on municipality are in parentheses. *** Significance at the 1 percent level. ** Significance at the 5 percent level. * Significance at the 10 percent level.

Data source: The SALAR.

Table 2: First-stage estimates when controlling for municipality fixed-effects.

\begin{tabular}{lcccc}
\hline & Full sample & $h=15$ & $h=10$ & $h=5$ \\
\hline $\bar{p}=1$ & $2.031^{* * *}$ & $1.923^{* * *}$ & $1.802^{* * *}$ & $1.467^{* * *}$ \\
& $(0.174)$ & $(0.181)$ & $(0.206)$ & $(0.257)$ \\
\hline $\bar{p}=2$ & $1.568^{* * *}$ & $1.294^{* * *}$ & & \\
& $(0.256)$ & $(0.367)$ & & \\
\hline $\bar{p}=3$ & $1.119^{* * *}$ & $1.198^{* * *}$ & & \\
& $(0.366)$ & $(0.399)$ & & \\
\hline Observations & 2511 & 2346 & 2047 & 1241 \\
\hline
\end{tabular}

Note: For different bandwidths, $h$, and order of polynomials, $\bar{p}$, the table reports estimates of $\alpha_{1}$ in the first-stage equation (3) on cost-equalizing grants, when controlling for municipality fixed-effects. Standard errors clustered on municipality are in parentheses. *** Significance at the 1 percent level. ** Significance at the 5 percent level. * Significance at the 10 percent level.

Data source: The SALAR. 
Table 3: Effects of grants on municipal personnel when controlling for municipality fixed-effects (2SLS estimates).

\begin{tabular}{|c|c|c|c|c|c|}
\hline & & Full sample & $h=15$ & $h=10$ & $h=5$ \\
\hline Total personnel & $\begin{array}{l}\bar{p}=1 \\
\bar{p}=2 \\
\bar{p}=3\end{array}$ & $\begin{array}{c}\mathbf{0 . 1 3 4}^{* * *} \\
(\mathbf{0 . 0 4 7 5 )} \\
-0.114 \\
(0.101) \\
-0.193 \\
(0.213)\end{array}$ & $\begin{array}{c}\mathbf{0 . 0 8 4 3} \\
(\mathbf{0 . 0 5 2 9}) \\
-0.156 \\
(0.175) \\
-0.123 \\
(0.201)\end{array}$ & $\begin{array}{c}0.0614 \\
(0.0625)\end{array}$ & $\begin{array}{c}0.0462 \\
(0.0956)\end{array}$ \\
\hline Administrative personnel & $\begin{array}{l}\bar{p}=1 \\
\bar{p}=2 \\
\bar{p}=3\end{array}$ & $\begin{array}{c}0.0133 \\
(0.00836) \\
0.0116 \\
(0.0156) \\
\mathbf{0 . 0 1 6 3} \\
(\mathbf{0 . 0 2 8 4})\end{array}$ & $\begin{array}{c}\mathbf{0 . 0 1 1 2} \\
(\mathbf{0 . 0 0 9 3 9 )} \\
0.0261 \\
(0.0248) \\
0.0333 \\
(0.0297)\end{array}$ & $\begin{array}{l}0.0176^{*} \\
(0.0106)\end{array}$ & $\begin{array}{c}0.0225 \\
(0.0160)\end{array}$ \\
\hline Child care personnel & $\begin{array}{l}\bar{p}=1 \\
\bar{p}=2 \\
\bar{p}=3\end{array}$ & $\begin{array}{c}\mathbf{0 . 0 0 2 5 2} \\
(\mathbf{0 . 0 1 3 7 )} \\
-0.0925^{* * *} \\
(0.0349) \\
-0.129^{*} \\
(0.0686)\end{array}$ & $\begin{array}{c}\mathbf{- 0 . 0 1 7 0} \\
(\mathbf{0 . 0 1 4 8 )} \\
-0.0897^{*} \\
(0.0495) \\
-0.0818 \\
(0.0545)\end{array}$ & $\begin{array}{c}-0.0375^{* *} \\
(0.0180)\end{array}$ & $\begin{array}{c}-0.0179 \\
(0.0288)\end{array}$ \\
\hline School personnel & $\begin{array}{l}\bar{p}=1 \\
\bar{p}=2 \\
\bar{p}=3\end{array}$ & $\begin{array}{c}\mathbf{0 . 0 4 0 2}^{* *} \\
(\mathbf{0 . 0 1 6 9 )} \\
0.0152 \\
(0.0332) \\
-0.00320 \\
(0.0673)\end{array}$ & $\begin{array}{c}\mathbf{0 . 0 3 7 2}^{* *} \\
(\mathbf{0 . 0 1 8 7}) \\
-0.0408 \\
(0.0620) \\
-0.0267 \\
(0.0681)\end{array}$ & $\begin{array}{l}0.0431^{*} \\
(0.0220)\end{array}$ & $\begin{array}{c}-0.0241 \\
(0.0426)\end{array}$ \\
\hline Elderly care personnel & $\begin{array}{l}\bar{p}=1 \\
\bar{p}=2 \\
\bar{p}=3\end{array}$ & $\begin{array}{c}0.0912^{* * *} \\
(0.0303) \\
\mathbf{0 . 0 1 2 1} \\
(\mathbf{0 . 0 5 7 8}) \\
0.00195 \\
(0.111)\end{array}$ & $\begin{array}{c}0.0767^{* *} \\
(0.0356) \\
\mathbf{0 . 0 3 3 7} \\
(\mathbf{0 . 0 9 9 2}) \\
0.0252 \\
(0.111)\end{array}$ & $\begin{array}{c}0.0680 \\
(0.0425)\end{array}$ & $\begin{array}{c}0.105^{*} \\
(0.0564)\end{array}$ \\
\hline Social welfare personnel & $\begin{array}{l}\bar{p}=1 \\
\bar{p}=2 \\
\bar{p}=3\end{array}$ & $\begin{array}{c}\mathbf{- 0 . 0 0 7 7 2} \\
(\mathbf{0 . 0 0 6 1 7}) \\
-0.0230^{*} \\
(0.0138) \\
-0.0376 \\
(0.0286)\end{array}$ & $\begin{array}{c}-\mathbf{- 0 . 0 1 1 7} \\
(\mathbf{0 . 0 0 7 0 1}) \\
-0.0323 \\
(0.0260) \\
-0.0287 \\
(0.0279)\end{array}$ & $\begin{array}{c}-0.0140 \\
(0.00976)\end{array}$ & $\begin{array}{l}-0.0190 \\
(0.0205)\end{array}$ \\
\hline Technical personnel & $\begin{array}{l}\bar{p}=1 \\
\bar{p}=2 \\
\bar{p}=3\end{array}$ & $\begin{array}{c}\mathbf{- 0 . 0 0 5 9 5} \\
\mathbf{( 0 . 0 1 5 0 )} \\
-0.0382 \\
(0.0296) \\
-0.0447 \\
(0.0636)\end{array}$ & $\begin{array}{c}\mathbf{- 0 . 0 1 2 3} \\
(\mathbf{0 . 0 1 7 1 )} \\
-0.0564 \\
(0.0578) \\
-0.0472 \\
(0.0666)\end{array}$ & $\begin{array}{l}-0.0161 \\
(0.0210)\end{array}$ & $\begin{array}{l}-0.0259 \\
(0.0345)\end{array}$ \\
\hline Observations & & 2511 & 2344 & 2045 & 1230 \\
\hline
\end{tabular}

Note: For different bandwidths, $h$, and order of polynomials, $\bar{p}$, the table reports estimates of $\beta_{1}$ in the secondstage equation (4) when controlling for municipality fixed-effects, with the dependent variables total personne as well as personnel disasegated by the different sectors. The AIC-preferred polynomial is in bold. Standard as werl the 5 percent level. * Significance at the 10 percent level.

Data source: The SALAR. 
Table 4: First-stage estimates using the shorter panel.

\begin{tabular}{lcccc}
\hline & Full sample & $h=15$ & $h=10$ & $h=5$ \\
\hline $\bar{p}=1$ & $4.449^{* * *}$ & $4.097^{* * *}$ & $4.308^{* * *}$ & 1.273 \\
& $(0.620)$ & $(0.750)$ & $(0.863)$ & $(1.170)$ \\
\hline $\bar{p}=2$ & $3.828^{* * *}$ & 2.914 & & \\
& $(0.799)$ & $(1.855)$ & & \\
\hline $\bar{p}=3$ & 2.527 & 2.845 & & \\
& $(1.911)$ & $(1.992)$ & & \\
\hline Observations & 837 & 803 & 691 & 385
\end{tabular}

Note: For different bandwidths, $h$, and order of polynomials, $\bar{p}$, the table reports estimates of $\alpha_{1}$ in the first-stage equation (3) on cost-equalizing grants. Standard errors clustered on municipality are in parentheses. *** Significance at the 1 percent level. ${ }^{* *}$ Significance at the 5 percent level. * Significance at the 10 percent level.

Data source: The Swedish Association of Local Authorities and Regions (SALAR).

Table 5: Effects of grants on municipal personnel using the shorter panel (2SLS estimates).

\begin{tabular}{lccc}
\hline & Full sample & $h=15$ & $h=10$ \\
\hline Total personnel & 0.0280 & -0.0998 & -0.0821 \\
& $(0.0583)$ & $(0.0784)$ & $(0.0858)$ \\
\hline Administrative personnel & $0.0251^{* * *}$ & $0.0276^{* * *}$ & $0.0401^{* * *}$ \\
& $(0.00551)$ & $(0.00788)$ & $(0.00909)$ \\
\hline Child care personnel & -0.00762 & $-0.0210^{*}$ & -0.0176 \\
& $(0.00781)$ & $(0.0112)$ & $(0.0144)$ \\
\hline School personnel & -0.0180 & $-0.0465^{*}$ & $-0.0558^{*}$ \\
& $(0.0171)$ & $(0.0260)$ & $(0.0325)$ \\
\hline Elderly care personnel & 0.0436 & -0.0285 & -0.0447 \\
& $(0.0314)$ & $(0.0381)$ & $(0.0468)$ \\
\hline Social welfare personnel & $-0.00965^{* *}$ & -0.00367 & -0.00256 \\
& $(0.00402)$ & $(0.00630)$ & $(0.00848)$ \\
\hline Technical personnel & -0.00547 & -0.0277 & -0.00152 \\
& $(0.0154)$ & $(0.0210)$ & $(0.0219)$ \\
\hline Observations & 837 & 803 & 691 \\
\hline
\end{tabular}

Note: For different bandwidths, $h$, and order of polynomial $\bar{p}=1$, the table reports estimates of $\beta_{1}$ in the second-stage equation (4), with the dependent variables total personnel as well as personnel disaggregated by the different sectors. Standard errors clustered on municipality are in parentheses. *** Significance at the 1 percent level. ${ }^{* *}$ Significance at the 5 percent level. * Significance at the 10 percent level.

Data source: The SALAR. 
Table 6: Effects of grants on private personnel in the local public welfare sector employed by non-profit or for-profit private firms (2SLS estimates).

\begin{tabular}{lccc}
\hline & Full sample & $h=15$ & $h=10$ \\
\hline Child care personnel & 0.0113 & $0.0261^{* * *}$ & 0.0170 \\
& $(0.00781)$ & $(0.00986)$ & $(0.0115)$ \\
\hline School personnel & $0.0267^{* *}$ & $0.0442^{* *}$ & 0.0327 \\
& $(0.0131)$ & $(0.0178)$ & $(0.0210)$ \\
\hline Elderly care personnel & -0.0134 & 0.0317 & 0.0327 \\
& $(0.0164)$ & $(0.0287)$ & $(0.0353)$ \\
\hline Social welfare personnel & 0.00396 & 0.00192 & -0.0161 \\
& $(0.00746)$ & $(0.0110)$ & $(0.0139)$ \\
\hline Observations & 834 & 800 & 688 \\
\hline
\end{tabular}

Note: For different bandwidths, $h$, and order of polynomial $\bar{p}=1$, the table reports estimates of $\beta_{1}$ in the second-stage equation (4), with the dependent variables private personnel in child care, schools, elderly care and social welfare. Standard errors clustered on municipality are in parentheses. ${ }^{* * *}$ Significance at the 1 percent level. ${ }^{* *}$ Significance at the 5 percent level. * Significance at the 10 percent level.

Data source: Statistics Sweden \& The SALAR.

Table 7: Effects of grants on private school personnel (2SLS estimates).

\begin{tabular}{lccccc}
\hline & & Full sample & $h=15$ & $h=10$ & $h=5$ \\
\hline School personnel & $\bar{p}=1$ & 0.0156 & 0.0187 & 0.0155 & -0.00761 \\
& & $(0.0100)$ & $(0.0115)$ & $(0.0119)$ & $(0.0177)$ \\
& $\bar{p}=2$ & 0.0199 & -0.00483 & & \\
& & $(0.0142)$ & $(0.0189)$ & & \\
& $\bar{p}=3$ & $\mathbf{0 . 0 1 3 7}$ & $\mathbf{- 0 . 0 0 2 9 6}$ & & \\
\hline Observations & & $\mathbf{( 0 . 0 1 6 8 )}$ & $\mathbf{( 0 . 0 1 3 3 )}$ & & 1234 \\
\hline
\end{tabular}

Note: For different bandwidths, $h$, and order of polynomials, $\bar{p}$, the table reports estimates of $\beta_{1}$ in the second-stage equation (4), with the dependent variable private school personnel. The AIC-preferred polynomial is in bold. Standard errors clustered on municipality are in parentheses. ${ }^{* * *}$ Significance at the 1 percent level. ${ }^{* *}$ Significance at the 5 percent level. * Significance at the 10 percent level.

Data source: Statistics Sweden \& The SALAR. 